\title{
AVALIAÇÃO DE DIFERENTES TIPOS DE ATMOSFERAS MODIFICADAS NA VIDA ÚTIL DE CARAMBOLAS MINIMAMENTE PROCESSADAS ${ }^{1}$
}

\author{
LEANDRO CAMARGO NEVES ${ }^{2}$, MARCOS ANDRÉ DE SOUZA PRILL ${ }^{3}$, VANUZA XAVIER DA SILVA4, \\ RONALDO MORENO BENEDETTE ${ }^{4}$, ROGÉRIO LOPES VIEITES 5
}

\begin{abstract}
RESUMO- Este trabalho teve por objetivo a avaliação da vida útil potencial de carambolas cv. Golden Star, minimamente processadas, armazenadas em diferentes tipos de embalagens plásticas, para a concepção da atmosfera modificada. Os frutos colhidos fisiologicamente maturos apresentaram coloração verde-amarelada, sólidos solúveis (SS) médios de 6,8 ${ }^{\circ}$ Brix e massa média de $185 \mathrm{~g}$. Antes da aplicação dos tratamentos, os frutos foram selecionados, higienizados em solução de hipoclorito de sódio ( $\mathrm{NaOCl}$ ) a $100 \mathrm{mg}$. $\mathrm{L}^{-1}$, resfriados por 12 horas a $15 \pm 0,5^{\circ} \mathrm{C}$, seccionados transversalmente, sendo então novamente higienizados em solução de $\mathrm{NaOCl}$ a 10 mg. $\mathrm{L}^{-1}$, por 3 minutos. Em seguida, os pedaços em forma de estrelas foram acondicionados em bandejas rígidas de poliestireno, com capacidade para $250 \mathrm{~g}$, e revestidas com os seguintes materiais: T1: filme plástico perfurado de polietileno de baixa densidade (PEBD), de 0,006 mm; T2: filme plástico poliolefínico com antiembassamento da Dupont ${ }^{\circledR}$ (AGF), de $0,015 \mathrm{~mm}$; T3: filme plástico poliolefínico da Dupont ${ }^{\circledR}(\mathrm{HF})$, de $0,015 \mathrm{~mm}$; T4: filme plástico de PEBD, de $60 \mu \mathrm{m}$; T5: filme plástico de PEBD, de $80 \mu \mathrm{m}$; T6: filme plástico de polipropileno (PP), de $22 \mu \mathrm{m}$, e T7: bandeja rígida de polietileno tereftalato (PET), com capacidade para $500 \mathrm{~mL}$, com tampa do mesmo material. As embalagens foram então armazenadas em câmara frigorífica a $12 \pm 0,5^{\circ} \mathrm{C}$ e $90 \pm 3 \%$ de U.R, por 18 dias. Observou-se que não houve diferença significativa quanto aos sólidos solúveis (SS) e acidez titulável (AT). O maior número de microrganismos e valor de $\mathrm{pH}$ foram observados nos frutos embalados nos filmes plásticos de PEBD de $0,006 \mathrm{~mm}$, AGF e HF de 0,015 mm da Dupont ${ }^{\circledR}$. Entretanto, os frutos acondicionados nas embalagens PET apresentaram o maior teor de ácido ascórbico. Da mesma forma, somente nas embalagens PET é que se conseguiu modificação atmosférica eficiente do ponto de vista da manutenção dos atributos de qualidade durante o AR. Assim, esse tratamento proporcionou adequado controle microbiológico e manutenção das características de qualidade por 18 dias para as carambolas minimamente processadas.
\end{abstract}

Palavras-chave: Averrhoa carambola, qualidade, conservação, embalagens plásticas

\section{EVALUATION OF DIFFERENT TYPES OF MODIFIED ATMOSPHERE IN COLD STORAGE OF STAR FRUITS MINIMALLY PROCESSED}

\begin{abstract}
This work aimed to evaluate the potential of cold storage of star fruits cv. Golden Star, minimally processed, by using different types of packing, for the analysis of the modified passive atmosphere. The fruits harvested in nature-green stage showed green-yellowish coloration, soluble solids (SS) an average of $6,8^{\circ}$ Brix and an average mass of $185 \mathrm{~g}$. Before the treatments were set, the fruits had been selected, cleaned in solution of $\mathrm{NaOCl}$ at $10 \mathrm{mg}$. $\mathrm{L}^{-1}$, cooled for 12 hours at $15 \pm 0,5^{\circ} \mathrm{C}$, cut transversally, and cleaned in solution of $\mathrm{NaOCl}$ at $10 \mathrm{mg}$. $\mathrm{L}^{-1}$, for 3 minutes. After, the pieces in form of stars had been displayed in rigid polystyrene trays, with capacity for $250 \mathrm{~g}$, and coated with the following materials: T1: perforated film plastic of low density polyethylene (LDPE), with 0,006 mm; T2: polyolephinic plastic film with anti-fog of DuPont ${ }^{\circledR}$ (AGF), with 0,015mm; T3: polyolephinic plastic film of Dupont ${ }^{\circledR}(\mathrm{HF})$, with 0,015 mm; T4: LDPE plastic film, with 0,060 $\mathrm{mm}$; T5: LDPE plastic film, with 0,080 mm; T6: polypropylene plastic film (PP), with 0,022 $\mathrm{mm}$ and T7: rigid polyethylene terephtalat tray (PET), with capacity for $500 \mathrm{~mL}$, with cover of the same material. The treatments had been conditioned in refrigerating chamber at $12 \pm 0,5^{\circ} \mathrm{C}$ and $90 \pm 3 \%$ of R.U., for 18 days. At the end, it was not observed significant difference in the tested treatments in the analysis of soluble solids (SS) and titratable acidity (TA). The biggest microbiological activity and the greater $\mathrm{pH}$ had been detected in the fruits packed in LDPE films with $6 \mu \mathrm{m}$, AGF and HF with $15 \mu \mathrm{m}$ from Dupont. However, the fruits conditioned in packing PET had shown the biggest contents of ascorbic acid. In the same way, only in PET packing was obtained an efficient atmospheric modification in the point of maintaining the quality of sensorial attributes during RA. Thus, this treatment provided the adequate microbiological control and maintenance of the sensorial attributes for 18 days for minimally processed star fruits.
\end{abstract}

Index-Terms: Averrhoa carambola, quality, shelf-life, plastic packages.

\section{INTRODUÇÃO}

O processamento mínimo de frutos e hortaliças tem como objetivo fornecer produtos com características semelhantes às de produtos frescos, sem comprometimento das qualidades nutricionais e com vida útil suficiente para sua distribuição, comercialização e consumo (Pereira et al., 2003). Outra vantagem desse tipo de produto é a drástica redução dos desperdícios, através do eficaz aproveitamento da matéria-prima (Junqueira e Luengo, 2000).

Apesar de sua praticidade e conveniência, o processamento mínimo pode proporcionar aos vegetais comportamento similar ao de tecidos submetidos a algum tipo de ferimento e/ou condições de estresse (Hong \& Kim, 2001), conduzindo a alterações bioquímicas e fisiológicas indesejáveis. A perda da integridade celular na superfície cortada dos frutos e hortaliças destrói a compartimentalização entre enzimas e substratos, levando ao escurecimento e à formação de metabólitos secundários indesejáveis. Com o aumento da respiração e da produção de etileno, podem ocorrer a aceleração da senescência e também a formação de sabores e odores desagradáveis. Outra limitação é resultante do exsudato da superfície cortada, que se torna um meio favorável ao crescimento de fungos e bactérias (Ahvenainen, 1996).

O desenvolvimento e a aceleração desses eventos metabólicos e microbiológicos estão relacionados às operações de descascamento e/ou seccionamento (Ahvenainen, 1996). Sendo assim, existe a necessidade de utilização de uma combinação de processos, como é o caso da desinfecção com soluções de hipoclorito de sódio ou sódio ativo (Pereira et al., 2003; Arruda et al., 2003), o

\footnotetext{
(Trabalho 195-2005). Recebido: 20-10-2005. Aceito para publicação: 29-09-2006.

${ }^{2}$ UFRR - Prof. Depto. de Fitotecnia - Centro de Ciências Agrárias, km 12 BR 174 s/nº, CEP 69301-970 - Boa Vista - RR. rapelbtu@ hotmail.com

${ }^{3}$ UFRR - Pós-Graduando em Ciência e Tecnologia de Alimentos - Centro de Ciências Agrárias, km 12 BR 174 s/nº, CEP 69301-970 - Boa Vista - RR. marcosprill@bol.com.br

${ }^{4}$ UFRR - Graduando em Agronomia - Centro de Ciências Agrárias, km 12 BR 174 s/nº, CEP 69301-970 - Boa Vista - RR. rmbenedette@ hotmail.com

${ }^{5}$ UNESP/FCA - Prof. Depto. de Gestão e Tecnologia Agroindustrial. C.P. 237, CEP 18603-970, Botucatu - SP. vieites@ fca.unesp.br
} 
uso de soluções de $\mathrm{CaCl}_{2}$ (Antoniolli et al., 2001), bem como a utilização de atmosferas modificadas (AM), através do uso de filmes plásticos (Pereira et al., 2003; Arruda et al., 2003), de modo a assegurar a qualidade e aumentar a vida de prateleira desses produtos.

Neste sentido, aproveitando-se ainda da inserção facilitada para produtos tropicais nos grandes mercados internacionais, como é o caso da carambola (Donadio, 2001), o presente trabalho tem por objetivo avaliar a influência da modificação atmosférica na vida útil de carambolas cv. Golden Star, minimamente processadas.

\section{MATERIAL E MÉTODOS}

Os frutos foram colhidos em propriedade agrícola situada na zona rural do município de Boa Vista-RR, localizada na Latitude $2^{\circ}$ $50^{\prime} 06^{\prime \prime} \mathrm{N}$ e Longitude $60^{\circ} 40^{\prime} 28^{\prime \prime} \mathrm{W}$, no estádio de fisiologicamente maturos, apresentando coloração verde-amarelada (70 e 30\%, respectivamente), sólidos solúveis (SS) médios de $6,8^{\circ} \mathrm{Brix}$, acidez titulável (AT) em média de 13,9 Cmol.L $\mathrm{L}^{-1}$ e massa média de $185 \mathrm{~g}$.

O trabalho foi conduzido no Centro de Ciências Agrárias (CCA), da Universidade Federal de Roraima (UFRR). Após a colheita, os frutos foram levados em veículo aberto até o Laboratório de Fitotecnia $\left(15 \pm 2^{\circ} \mathrm{Ce} 75 \pm 3 \%\right.$ de U.R.), dentro de contentores de 20 $\mathrm{kg}$, sendo então selecionados pela ausência de danos e/ou podridões e higienizados em solução de hipoclorito de sódio $(\mathrm{NaOCl})$ a 100 mg.L $\mathrm{L}^{-1}$, por 10 minutos. Realizado o enxágüe e a secagem em bandejas perfuradas expostas ao ar atmosférico, os frutos foram resfriados em B.O.D. por $10 \mathrm{~h}$ a $15 \pm 0,5^{\circ} \mathrm{C}$, visando à desaceleração metabólica. Para a constituição dos tratamentos, os frutos foram seccionados transversalmente, na forma de estrela, em pedaços de $2,5 \pm 0,5 \mathrm{~cm}$, por $7 \pm 1,5 \mathrm{~cm}$ de área, sendo então novamente higienizados em solução de $\mathrm{NaOCl}$ a $10 \mathrm{mg} . \mathrm{L}^{-1}$, por 3 minutos. Após o processamento, os pedaços foram acondicionados em bandejas rígidas de poliestireno, com capacidade para $250 \mathrm{~g}$, as quais foram revestidas com sacolas plásticas de $0,2 \times 0,25 \mathrm{~m}$ dos seguintes materiais: T1: filme plástico perfurado de polietileno de baixa densidade (PEBD), de $0,006 \mathrm{~mm}\left(\mathrm{TPO}_{2}=18.083 \mathrm{~cm}^{3} \cdot \mathrm{m}^{-2} \cdot \mathrm{dia}^{-1}\right.$, a $25^{\circ} \mathrm{C}$ e $1 \mathrm{~atm}$; $\mathrm{TPCO}_{2}=75.552 \mathrm{~cm}^{3} \cdot \mathrm{m}^{-2} \cdot \mathrm{dia}^{2}$, a $25{ }^{\circ} \mathrm{C}$ e $1 \mathrm{~atm}$; área de permeação $\left.=790 \mathrm{~cm}^{2}\right) ; \mathrm{T} 2$ : filme plástico poliolefínico com antiembassamento da Dupont ${ }^{\circledR}(\mathrm{AGF})$, de $0,015 \mathrm{~mm}\left(\mathrm{TPO}_{2}=11.850\right.$, $\mathrm{TPCO}_{2}=43.212$, área de permeação $\left.=700 \mathrm{~cm}^{2}\right)$; T3: filme plástico polioefínico da Dupont ${ }^{\circledR}(\mathrm{HF})$, de $0,015 \mathrm{~mm}\left(\mathrm{TPO}_{2}=10.116, \mathrm{TPCO}_{2}\right.$ $=38.633$, área de permeação $\left.=780 \mathrm{~cm}^{2}\right) ;$ T4: filme plástico de PEBD, de $0,060 \mathrm{~mm}\left(\mathrm{TPO}_{2}=3.565, \mathrm{TPCO}_{2}=13.888\right.$, área de permeabilidade $\left.=920 \mathrm{~cm}^{2}\right)$; T5: filme plástico de PEBD, de $0,080 \mathrm{~mm}\left(\mathrm{TPO}_{2}=2.302\right.$, $\mathrm{TPCO}_{2}=8.756$, área de permeabilidade $\left.=990 \mathrm{~cm}^{2}\right)$; T6: filme plástico de polipropileno (PP), de 0,022 $\mathrm{mm}\left(\mathrm{TPO}_{2}=4.842, \mathrm{TPCO}_{2}=9.105\right.$, área de permeabilidade $\left.=810 \mathrm{~cm}^{2}\right)$, e T7: bandeja rígida de polietileno tereftalato (PET), com capacidade para $500 \mathrm{~mL}$, com tampa do mesmo material $\left(\mathrm{TPO}_{2}=12.892\right.$ e área de permeação $\left.=370 \mathrm{~cm}^{2}\right)$. A seguir, as bandejas foram acondicionadas em câmara frigorífica a $12 \pm 0,5^{\circ} \mathrm{Ce}$ $95 \pm 3 \%$ de U.R, por 18 dias e, durante esse período, foram analisados nos tempos $1 ; 2 ; 3 ; 5 ; 7 ; 9 ; 12 ; 15$ e 18 dias de armazenamento refrigerado (AR) quanto aos teores de sólidos solúveis (SS), determinados através da leitura refratométrica direta, com o refratômetro tipo Abbe, marca ATAGO - N1, resultados expressos em ${ }^{\circ}$ Brix; acidez titulável (AT), determinada através de titulometria de neutralização ( $\mathrm{NaOH}$ a $0,01 \mathrm{n}$ ), com ponto de viragem no $\mathrm{pH} 8,2 \mathrm{e}$ resultados expressos em Cmol. $\mathrm{L}^{-1} ; \mathrm{pH}$, determinado através de potenciometria em amostra triturada e homogeneizada; ocorrência de desordens fisiológicas nos frutos seccionados, avaliada através da porcentagem média desses danos em cada embalagem (20 repetições de cada tratamento), utilizando-se de um índice de aceitabilidade máximo de não mais do que $25 \%$ dos frutos de cada embalagem apresentando pigmentação não-característica; teor de ácido ascórbico (mg por $100 \mathrm{~g}$ de polpa), determinado segundo método de Carvalho et al. (1990), que se baseia na redução do indicador 2,6 - diclorobenzenoindolfenol (DCFI) pelo ácido ascórbico; desenvolvimento de fungos, leveduras, coliformes totais/ fecais, Salmonella e bactérias lácticas, segundo metodologia proposta por Vanderzante \& Splittstoesser (1992), obedecendo à Resolução RCD n ${ }^{\circ} 12$, de 2 de janeiro de 2001, da Agência Nacional de Vigilância Sanitária (ANVISA). Também avaliou-se a composição gasosa presente no espaço livre das embalagens, utilizando cromatógrafo gasoso marca Varian, modelo 3300, sendo os resultados expressos em porcentagem. Quantificou-se, ainda, a taxa de permeabilidade dos materiais de embalagem, através do método de aumento da concentração, segundo protocolo citado por Arruda et al. (2003) e descrito por Oliveira et al. (1996), sendo os resultados expressos em $\mathrm{cm}^{3} \cdot \mathrm{m}^{2} \cdot \mathrm{dia}^{-1}$.

O delineamento experimental empregado foi o inteiramente casualizado, seguindo um esquema fatorial 7x9 (7 embalagens e 9 tempos de AR), com 20 repetições para análise de desordens fisiológicas (contendo uma amostra por repetição) e 5 repetições para as demais análises (contendo 3 amostras - embalagens, por repetição). Os dados foram submetidos à análise de variância e à comparação de médias, pelo teste de Tukey (pd"0,05).

\section{RESULTADOS E DISCUSSÃO}

Composição Gasosa das Embalagens - Com relação à composição gasosa do espaço livre das embalagens, puderam-se detectar 4 comportamentos distintos (Figura 1). Primeiramente, observou-se, nas embalagens onde as concentrações gasosas permaneceram constantes e equilibradas com o ar atmosférico, que praticamente não houve relação entre o avanço do amadurecimento dos pedaços de carambola e as embalagens plásticas (Figura 1a). Nas embalagens de PEBD de 0,006 mm perfuradas, AGF e HF de 0,015 mm, não foram detectadas modificações expressivas na concentração de $\mathrm{O}_{2}$ no interior das embalagens, quando comparada com a concentração de $\mathrm{O}_{2}$ do meio externo. Esses tratamentos apresentaram concentrações estáveis e constantes, variando entre $21,87 \pm 1 \%$ no primeiro dia de análise e $21,71 \pm 1 \%$ no último dia de AR. Quanto à concentração de $\mathrm{CO}_{2}$ nessas embalagens, pôde-se detectar o mesmo comportamento. No primeiro dia de avaliação, constataram-se variações entre $0,92 \pm$ $0,04 \%$, ligeiro acréscimo aos 12 dias de $\mathrm{AR}$, atingindo valores entre $0,95 \pm 0,06 \%$, o que seguramente não se pode relacionar a nenhum pico climatérico de respiração, pois, caracteristicamente, carambolas não apresentam comportamento de frutos climatéricos (Neves et al., 2004). Aos 18 dias de AR, detectaram-se concentrações de $\mathrm{CO}_{2}$ variando entre $0,88 \pm 0,07 \%$, o que confirma o diagnóstico de uma sequiência de dados relativamente linear e sem modificações expressivas. Da mesma forma, as taxas de permeabilidade desses filmes foram relativamente altas, superior a $10.000 \mathrm{~cm}^{3} \cdot \mathrm{m}^{2} \cdot \mathrm{dia}^{-1}$ para $\mathrm{O}_{2}$ e superior a $38.000 \mathrm{~cm}^{3} \cdot \mathrm{m}^{2} \cdot \mathrm{dia}^{-1}$ para $\mathrm{CO}_{2}$, explicando, assim, a baixa eficiência na modificação da atmosfera gasosa dessas embalagens.

Um segundo tipo de comportamento refere-se às embalagens plásticas, onde, mesmo havendo modificações na composição gasosa do interior das embalagens, não se conseguiu atingir as concentrações sugeridas por Cantwell (1992), ou seja, de 2 a $8 \%$ de $\mathrm{O}_{2}$ e de 5 a $15 \%$ de $\mathrm{CO}_{2}$, para que, segundo o mesmo, ocorresse a melhor conservação de frutos minimamente processados (Figura 1b). Nas embalagens plásticas de PP, de $0,022 \mathrm{~mm}$, observou-se suave decréscimo na concentração de $\mathrm{O}_{2}$ ao longo do período experimental. Assim, no primeiro dia de avaliação, observaram-se valores médios de 20,62 \%, chegando ao final dos 18 dias de AR com valores médios de $17,10 \%$. Essas concentrações são consideradas como insuficientes para a manutenção da qualidade sensorial de produtos minimamente processados. Os níveis de $\mathrm{CO}_{2}$ detectados nesse tratamento alcançaram concentrações médias de 2,34\% no início do experimento e 3,96\% aos 18 dias de AR. Semelhantemente, essas condições também podem ser prescritas 

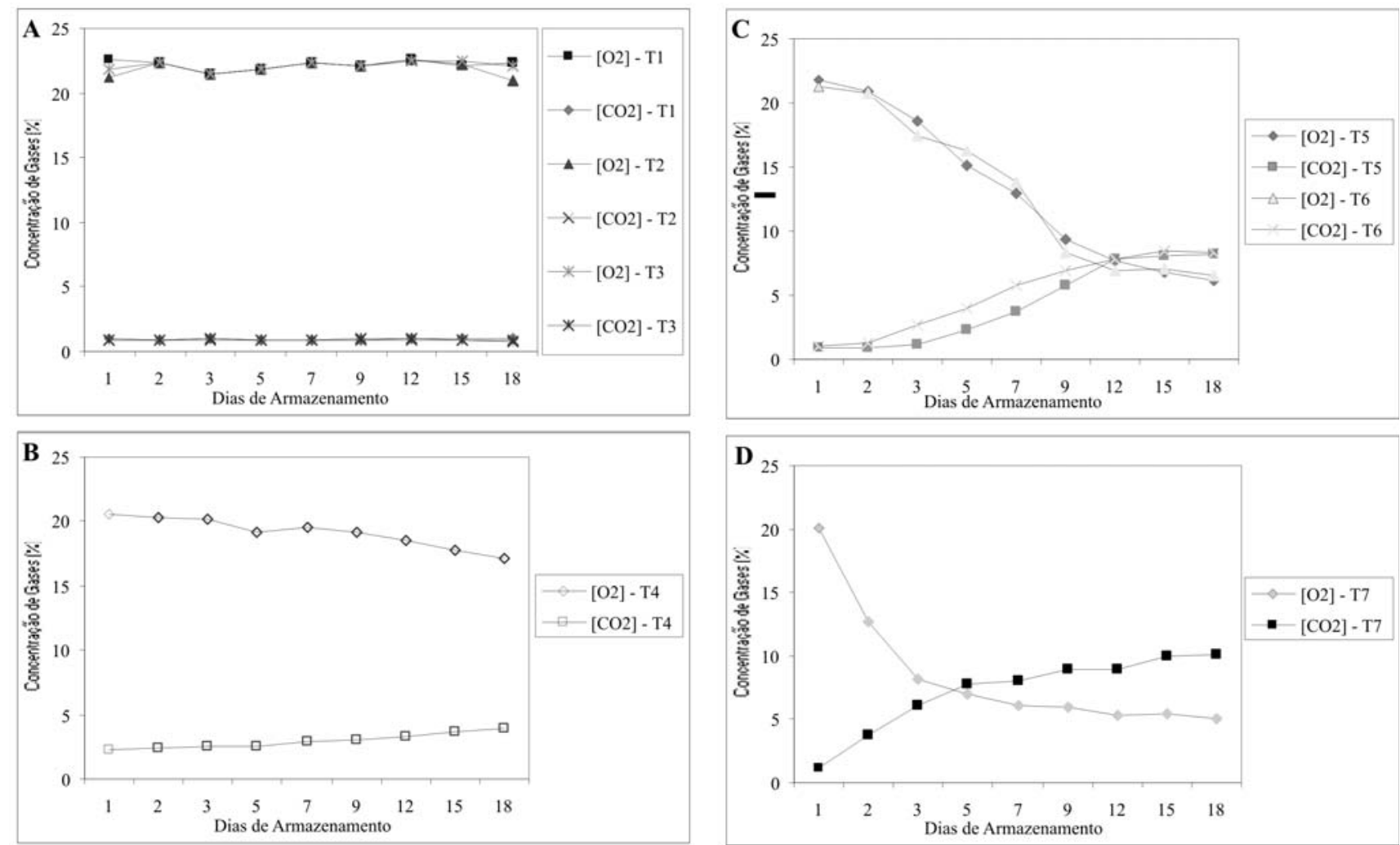

FIGURA 1 - Composição gasosa do espaço livre das embalagens acondicionando carambolas cv. Golden Star, minimamente processadas e frigoarmazenadas por 18 dias a $12 \pm 0,5^{\circ} \mathrm{C}$ e U.R. $95 \pm 5 \%$. A - PEBD de $0,006 \mathrm{~mm}$ perfurado, AGF da Dupont ${ }^{\circ}$ de $0,015 \mathrm{~mm}$ e HF da Dupont ${ }^{\circledR}$ de $0,015 \mathrm{~mm} ; \mathbf{B}$ - PP de 0,022mm; C - PEBD de 0,060 mm e PEBD de 0,080 mm; D - PET. Não existe diferença estatística significativa dentro de cada figura.

como insuficientes para o controle dos eventos que estimulam o avanço do amadurecimento (Brackmann et al., 2003; Lana \& Finger, 2000; Cantwell, 1992), bem como qualquer menção de controle microbiológico dos frutos, dada a reconhecida ação fúngica promovida pela elevação dos níveis de $\mathrm{CO}_{2}$ (Donazollo et al., 2003). Sendo assim, é possível relacionar a baixa eficiência do controle atmosférico interno nas embalagens, observada nos filmes de PEBD de $0,006 \mathrm{~mm}$ perfurados, AGF e HF de $0,015 \mathrm{~mm}$, e PP de $0,022 \mathrm{~mm}$, com os elevados índices de contaminação microbiológica detectados nesses tratamentos (Tabelas 2 e 3 ).

Foram observados, também, tratamentos que apresentaram diminuição da concentração de $\mathrm{O}_{2}$ e manutenção de níveis satisfatórios de $\mathrm{CO}_{2}$ tardiamente, ou seja, somente após o terço final do período experimental (Figura 1c). Esse fato, por sua vez, não contribuiu para o controle efetivo do desenvolvimento de contaminantes microbiológicos. Foi o caso dos pedaços de carambolas acondicionados nos filmes de PEBD de 0,060 e 0,080 $\mathrm{mm}$, onde, inicialmente, os níveis de $\mathrm{O}_{2}$ estavam entre $21,52 \pm 0,27 \%$ e reduziram para concentrações entre $7,33 \pm 0,38 \%$ aos 12 dias de $\mathrm{AR}$, chegando ao final do experimento com concentrações entre $6,35 \pm 0,18 \%$. Da mesma forma, para os níveis de $\mathrm{CO}_{2}$, onde somente aos 12 dias de AR foram constatadas concentrações médias superiores a 8,0\%, consideradas por Brackmann et al. (2003) como suficientes para um controle da velocidade metabólica e do desenvolvimento de microrganismos. Este fato comprova os resultados obtidos nesse experimento, em que os pedaços de carambola embalados nos filmes de PEBD de 0,060 e 0,080 mm se de se apresentaram ao final do período experimental com algum comprometimento microbiológico (Tabelas 3 e 4). Vale ainda ressaltar que os filmes de PEBD de 0,060 e 0,080 mm apresentaram baixa permeabilidade gasosa quando comparados aos tratamentos submetidos aos filmes plásticos de PEBD de 0,006 mm, AGF e HF de $0,015 \mathrm{~mm}$. Baseado nisso, dever-se-ia esperar maior modificação da atmosfera gasosa no interior das embalagens, influenciando desta forma nos resultados e, conseqüentemente, melhorando a manutenção da qualidade final, o que não foi observado. Provavelmente, a atividade metabólica da carambola minimamente processada exija barreiras mais eficientes quanto à diminuição das trocas gasosas com o meio exterior.

De acordo com a Figura 1d, podemos destacar o tratamento utilizando embalagens PET como aquele que, logo nos primeiros dias de AR, alcançou o efetivo estabelecimento da concentração atmosférica adequada segundo Cantwell (1992). Como conseqüência disso, a velocidade dos processos que desencadeiam a senescência foi reduzida, proporcionando, assim, a extensão do período de vida útil do produto. Neste sentido, as embalagens PET apresentavam, já no quinto dia de AR, concentrações médias de $\mathrm{O}_{2}$ de 6,98\%, estabilizando em valores médios de 5,21 $\pm 0,19 \%$ até o final do experimento. Constatou-se, então, a partir desses resultados, excelente controle no suprimento de $\mathrm{O}_{2}$ disponível no interior das embalagens PET, sem, contudo, incitar um comportamento de anaerobiose nos frutos. Os níveis de $\mathrm{CO}_{2}$, também aos cinco dias de AR, já se apresentavam com valores médios de 7,81\% e estabilizaram, na média, com 10,02\% dos 15 aos 18 dias de AR. Esses resultados refletiram diretamente na manutenção dos atributos qualitativos. Nesse sentido, os frutos acondicionados nas embalagens PET, não apresentavam sintomas de podridão e/ou contaminação microbiológica sem escurecimento dos pedaços de carambola. Por outro lado, esse tratamento apresentou maiores níveis de vitamina $\mathrm{C}$, proporcionando,assim, maior potencial comercial.

Contagem Microbiológica - Em todas as amostras analisadas, os níveis de Salmonella e de coliformes totais/fecais encontravam-se dentro dos padrões microbiológicos estabelecidos pela ANVISA (2001). Resultados semelhantes foram descritos por Pereira et al. (2003), trabalhando com goiabas var. Paloma e Bonnas et al. (2003), trabalhando com abacaxis cv. Smooth Cayenne, onde a sanificação com o $\mathrm{NaOCl}$ e a modificação atmosférica contribuíram para o eficiente controle de tais contaminantes patogênicos. Baseado nos resultados acima descritos, acredita-se que as condições higiênico- 
TABELA 1 - Sólidos solúveis, acidez titulável, pH e ácido ascórbico, em carambolas cv. Golden Star, minimamente processadas e frigoarmazenadas por 18 dias a $12 \pm 0,5^{\circ}$ C e U.R. $95 \pm 5 \%$.

\begin{tabular}{|c|c|c|c|c|c|c|c|c|c|c|}
\hline \multirow{2}{*}{ Variáveis } & \multirow[t]{2}{*}{ Dias } & \multicolumn{9}{|c|}{ Tratamentos * } \\
\hline & & T1 & T2 & T3 & T4 & T5 & T6 & T7 & D.M.S. & C.V. \\
\hline \multirow{9}{*}{$\begin{array}{l}\text { Sólidos } \\
\text { solúveis } \\
\text { ( }{ }^{\circ} \text { Brix) }\end{array}$} & 1 & $6,8 \mathrm{Aa}$ & 6,9Aa & $7,0 \mathrm{Aa}$ & $6,8 \mathrm{Aa}$ & $6,8 \mathrm{Aa}$ & 6,9Aa & 6,9Aa & 0,553 & 2,42 \\
\hline & 2 & $7,0 \mathrm{Aa}$ & $7,1 \mathrm{Aa}$ & $7,2 \mathrm{Aa}$ & $6,9 \mathrm{Aa}$ & $6,9 \mathrm{Aa}$ & $7,2 \mathrm{Aa}$ & $6,9 \mathrm{Aa}$ & 0,253 & 3,39 \\
\hline & 3 & $7,2 \mathrm{Aa}$ & $7,3 \mathrm{Aa}$ & $7,2 \mathrm{Aa}$ & $6,9 \mathrm{Aa}$ & $7,0 \mathrm{Aa}$ & $7,0 \mathrm{Aa}$ & $7,0 \mathrm{Aa}$ & 0,336 & 2,87 \\
\hline & 5 & $7,2 \mathrm{Aa}$ & $7,5 \mathrm{Aa}$ & $7,2 \mathrm{Aa}$ & $7,2 \mathrm{Aa}$ & $7,2 \mathrm{Aa}$ & $7,2 \mathrm{Aa}$ & $7,2 \mathrm{Aa}$ & 0,541 & 2,10 \\
\hline & 7 & $7,6 \mathrm{Aa}$ & $7,2 \mathrm{Aa}$ & 7,4Aa & $7,3 \mathrm{Aa}$ & $7,3 \mathrm{Aa}$ & $7,3 \mathrm{Aa}$ & $7,2 \mathrm{Aa}$ & 0,694 & 4,49 \\
\hline & 9 & $7,5 \mathrm{Aa}$ & $7,8 \mathrm{Aa}$ & $7,5 \mathrm{Aa}$ & 7,3Aa & $7,2 \mathrm{Aa}$ & $7,5 \mathrm{Aa}$ & $7,5 \mathrm{Aa}$ & 0,746 & 2,55 \\
\hline & 12 & 7,9Aa & $7,6 \mathrm{Aa}$ & 7,3Aa & $7,5 \mathrm{Aa}$ & $7,5 \mathrm{Aa}$ & $7,6 \mathrm{Aa}$ & $7,6 \mathrm{Aa}$ & 0,998 & 3,71 \\
\hline & 15 & $8,0 \mathrm{Aa}$ & 7,9Aa & 7,7Aa & 7,7Aa & $7,6 \mathrm{Aa}$ & 7,9Aa & $7,6 \mathrm{Aa}$ & 0,591 & 4,01 \\
\hline & 18 & $8,1 \mathrm{Aa}$ & 7,9Aa & 7,9Aa & $7,8 \mathrm{Aa}$ & 7,9Aa & $7,8 \mathrm{Aa}$ & $7,7 \mathrm{Aa}$ & 0,499 & 2,18 \\
\hline \multirow{9}{*}{$\begin{array}{c}\text { Acidez } \\
\text { titulável } \\
\left(\text { Cmol.L }^{-1}\right)\end{array}$} & 1 & $13,7 \mathrm{Aa}$ & $14,1 \mathrm{Aa}$ & $14,0 \mathrm{Aa}$ & $13,9 \mathrm{Aa}$ & $13,8 \mathrm{Aa}$ & $13,9 \mathrm{Aa}$ & $14,0 \mathrm{Aa}$ & 0,981 & 3,14 \\
\hline & 2 & $13,22 \mathrm{Aa}$ & $13,18 \mathrm{Aa}$ & $13,20 \mathrm{Aa}$ & $13,19 \mathrm{Aa}$ & $13,14 \mathrm{Aa}$ & $13,32 \mathrm{Aa}$ & $13,55 \mathrm{Aa}$ & 0,420 & 3,01 \\
\hline & 3 & $12,04 \mathrm{Aa}$ & $12,12 \mathrm{Aa}$ & $12,19 \mathrm{Aa}$ & $12,35 \mathrm{Aa}$ & $12,28 \mathrm{Aa}$ & $12,25 \mathrm{Aa}$ & $12,38 \mathrm{Aa}$ & 0,511 & 7,71 \\
\hline & 5 & $11,80 \mathrm{Aa}$ & $11,84 \mathrm{Aa}$ & $11,86 \mathrm{Aa}$ & $11,82 \mathrm{Aa}$ & $11,96 \mathrm{Aa}$ & $11,90 \mathrm{Aa}$ & $12,01 \mathrm{Aa}$ & 0,388 & 3,64 \\
\hline & 7 & $10,62 \mathrm{Aa}$ & $10,65 \mathrm{Aa}$ & $10,69 \mathrm{Aa}$ & $10,62 \mathrm{Aa}$ & $10,71 \mathrm{Aa}$ & $10,77 \mathrm{Aa}$ & $10,74 \mathrm{Aa}$ & 0,416 & 4,93 \\
\hline & 9 & $9,24 \mathrm{Aa}$ & $9,60 \mathrm{Aa}$ & $9,34 \mathrm{Aa}$ & $9,46 \mathrm{Aa}$ & $9,74 \mathrm{Aa}$ & $9,86 \mathrm{Aa}$ & $9,96 \mathrm{Aa}$ & 0,876 & 3,44 \\
\hline & 12 & $8,94 \mathrm{Aa}$ & $8,99 \mathrm{Aa}$ & $8,84 \mathrm{Aa}$ & $8,95 \mathrm{Aa}$ & $9,00 \mathrm{Aa}$ & $9,01 \mathrm{Aa}$ & $9,12 \mathrm{Aa}$ & 0,941 & 3,87 \\
\hline & 15 & $7,12 \mathrm{Aa}$ & $7,32 \mathrm{Aa}$ & $7,21 \mathrm{Aa}$ & $7,18 \mathrm{Aa}$ & $7,24 \mathrm{Aa}$ & $7,26 \mathrm{Aa}$ & $7,32 \mathrm{Aa}$ & 0,450 & 4,08 \\
\hline & 18 & $6,35 \mathrm{Aa}$ & $6,70 \mathrm{Aa}$ & $6,55 \mathrm{Aa}$ & $6,90 \mathrm{Aa}$ & $6,80 \mathrm{Aa}$ & $7,05 \mathrm{Aa}$ & $7,25 \mathrm{Aa}$ & 1,084 & 5,10 \\
\hline \multirow{9}{*}{ pH } & 1 & $3,62 \mathrm{Ca}$ & $3,56 \mathrm{Da}$ & $3,59 \mathrm{Ea}$ & $3,53 \mathrm{Da}$ & $3,61 \mathrm{Da}$ & $3,53 \mathrm{Da}$ & $3,55 \mathrm{Ba}$ & 0,102 & 2,94 \\
\hline & 2 & $4,06 \mathrm{Ca}$ & $3,99 \mathrm{Da}$ & $4,08 \mathrm{Ea}$ & $3,89 \mathrm{Db}$ & $3,79 \mathrm{Db}$ & $3,62 \mathrm{Db}$ & $3,67 \mathrm{Bb}$ & 0,343 & 5,02 \\
\hline & 3 & $4,59 \mathrm{Ca}$ & $4,42 \mathrm{Cb}$ & $4,49 \mathrm{Ea}$ & $4,06 \mathrm{Db}$ & $3,94 \mathrm{Db}$ & $3,78 \mathrm{Db}$ & $3,84 \mathrm{Bb}$ & 0,613 & 5,88 \\
\hline & 5 & $5,09 \mathrm{Ba}$ & $4,95 \mathrm{Ca}$ & $4,90 \mathrm{Da}$ & $4,16 \mathrm{Cb}$ & $4,12 \mathrm{Cb}$ & $4,01 \mathrm{Db}$ & $3,91 \mathrm{Bb}$ & 0,551 & 4,72 \\
\hline & 7 & $5,46 \mathrm{Ba}$ & $5,39 \mathrm{Ca}$ & $5,51 \mathrm{Da}$ & $4,55 \mathrm{Cb}$ & $4,49 \mathrm{Cb}$ & $4,26 \mathrm{Cb}$ & $3,98 \mathrm{Bb}$ & 0,600 & 4,92 \\
\hline & 9 & $5,98 \mathrm{Ba}$ & $5,76 \mathrm{Ba}$ & $5,86 \mathrm{Ca}$ & $4,76 \mathrm{Cb}$ & $4,81 \mathrm{Cb}$ & $4,49 \mathrm{Cb}$ & $4,03 \mathrm{Bc}$ & 0,385 & 4,48 \\
\hline & 12 & $6,43 \mathrm{Aa}$ & $6,03 \mathrm{Ba}$ & $5,99 \mathrm{Ca}$ & $4,99 \mathrm{Cb}$ & $4,86 \mathrm{Cb}$ & $4,61 \mathrm{Cb}$ & $4,14 \mathrm{Ac}$ & 0,571 & 6,11 \\
\hline & 15 & 6,91Aa & $6,84 \mathrm{Ba}$ & $6,49 \mathrm{Ba}$ & $5,82 \mathrm{Bb}$ & $5,43 \mathrm{Bb}$ & $5,25 \mathrm{Bb}$ & $4,22 \mathrm{Ac}$ & 0,632 & 5,42 \\
\hline & 18 & 7,20Aa & $7,24 \mathrm{Aa}$ & $7,19 \mathrm{Aa}$ & $6,91 \mathrm{Ab}$ & $6,93 \mathrm{Ab}$ & $6,91 \mathrm{Ab}$ & $4,26 \mathrm{Ac}$ & 0,225 & 5,10 \\
\hline \multirow{9}{*}{$\begin{array}{c}\text { Ácido } \\
\text { Ascórbico } \\
\text { (mg por } \\
\mathbf{1 0 0} \text { g de } \\
\text { polpa) }\end{array}$} & 1 & $15,22 \mathrm{Ab}$ & $17,89 \mathrm{Aa}$ & $18,02 \mathrm{Aa}$ & $18,12 \mathrm{Aa}$ & $17,45 \mathrm{Aa}$ & $18,22 \mathrm{Aa}$ & $18,95 \mathrm{Aa}$ & 1,752 & 9,95 \\
\hline & 2 & $10,84 \mathrm{Bb}$ & $12,14 \mathrm{Bb}$ & $11,65 \mathrm{Bb}$ & $13,20 \mathrm{Ba}$ & $13,96 \mathrm{Ba}$ & $14,21 \mathrm{Aa}$ & $15,22 \mathrm{Aa}$ & 3,984 & 10,12 \\
\hline & 3 & $3,44 \mathrm{Cb}$ & $5,74 \mathrm{Cb}$ & $4,49 \mathrm{Cb}$ & $9,10 \mathrm{Bb}$ & $9,94 \mathrm{Bb}$ & $10,96 \mathrm{Ba}$ & $13,36 \mathrm{Ba}$ & 2,442 & 8,40 \\
\hline & 5 & $0,54 \mathrm{Dc}$ & $1,16 \mathrm{Dc}$ & $1,01 \mathrm{Dc}$ & $6,65 \mathrm{Cb}$ & $7,01 \mathrm{Cb}$ & $7,42 \mathrm{Ca}$ & $10,84 \mathrm{Ba}$ & 3,851 & 12,16 \\
\hline & 7 & $0,78 \mathrm{Dc}$ & - & $0,38 \mathrm{Ec}$ & $3,31 \mathrm{Db}$ & $2,87 \mathrm{Db}$ & $2,29 \mathrm{Db}$ & $7,75 \mathrm{Ca}$ & 1,230 & 8,93 \\
\hline & 9 & - & - & $0,05 \mathrm{~Eb}$ & $0,91 \mathrm{~Eb}$ & $1,45 \mathrm{Db}$ & $1,09 \mathrm{~Eb}$ & $6,62 \mathrm{Ca}$ & 2,798 & 7,79 \\
\hline & 12 & - & - & - & $0,45 \mathrm{~Eb}$ & $0,94 \mathrm{~Eb}$ & $0,85 \mathrm{~Eb}$ & $3,91 \mathrm{Da}$ & 2,146 & 14,17 \\
\hline & 15 & - & - & ـ & $0,18 \mathrm{Fb}$ & $0,11 \mathrm{~Eb}$ & $0,54 \mathrm{~Eb}$ & $3,21 \mathrm{Da}$ & 2,390 & 12,99 \\
\hline & 18 & - & - & - & $0,08 \mathrm{Fb}$ & - & $0,37 \mathrm{~Eb}$ & $2,95 \mathrm{Da}$ & 2,443 & 15,02 \\
\hline
\end{tabular}

As médias seguidas das mesmas letras, nas linhas e nas colunas, não diferem entre si, ao nível de 5\% de probabilidade, pelo teste de Tukey.

*T1: PEBD de 6mm; T2: AGF da Dupont de 15mm; T3: HF da Dupont de 15mm; T4: PP de 22mm.; T5: PEBD de 60mm; T6: PEBD de 80mm.; T7: PET.

TABELA 2 - Contagem de fungos e leveduras, em carambolas cv. Golden Star, minimamente processadas e frigoarmazenadas por 18 dias a $12 \pm 0,5^{\circ}$ C e U.R. $95 \pm 5 \%$.

\begin{tabular}{cccccccc}
\hline Dias & \multicolumn{7}{c}{${ }^{* *}$ Tratamentos } \\
\hline & $\mathbf{T 1}$ & $\mathbf{T 2}$ & $\mathbf{T 3}$ & $\mathbf{T 4}$ & $\mathbf{T 5}$ & $\mathbf{T} \mathbf{6}$ & T7 \\
\hline $\mathbf{1}$ & $7,24 \times 10^{1} \mathrm{Fa}^{*}$ & $3,65 \times 10^{1} \mathrm{Fa}$ & $5,32 \times 10^{1} \mathrm{Ea}$ & $1,12 \times 10^{1} \mathrm{Da}$ & $1,21 \times 10^{-3} \mathrm{Bb}$ & $1,54 \times 10^{-3} \mathrm{Bb}$ & - \\
$\mathbf{2}$ & $3,71 \times 10^{2} \mathrm{Ea}$ & $4,18 \times 10^{1} \mathrm{Fa}$ & $7,12 \times 10^{1} \mathrm{Ea}$ & $5,24 \times 10^{1} \mathrm{Da}$ & $2,82 \times 10^{-3} \mathrm{Bb}$ & $1,93 \times 10^{-3} \mathrm{Bb}$ & - \\
$\mathbf{3}$ & $9,24 \times 10^{2} \mathrm{Ea}$ & $8,01 \times 10^{2} \mathrm{Ea}$ & $9,05 \times 10^{2} \mathrm{Da}$ & $9,24 \times 10^{1} \mathrm{Da}$ & $2,20 \times 10^{-3} \mathrm{Bb}$ & $3,54 \times 10^{-3} \mathrm{Bb}$ & - \\
$\mathbf{5}$ & $1,24 \times 10^{3} \mathrm{Da}$ & $9,70 \times 10^{2} \mathrm{Ea}$ & $1,02 \times 10^{3} \mathrm{Ca}$ & $3,77 \times 10^{2} \mathrm{Ca}$ & $5,21 \times 10^{-3} \mathrm{Bc}$ & $6,33 \times 10^{-3} \mathrm{Bc}$ & - \\
$\mathbf{7}$ & $5,18 \times 10^{4} \mathrm{Ca}$ & $2,12 \times 10^{3} \mathrm{Da}$ & $4,16 \times 10^{3} \mathrm{Ca}$ & $9,01 \times 10^{2} \mathrm{Ca}$ & $7,86 \times 10^{-3} \mathrm{Bc}$ & $7,54 \times 10^{-3} \mathrm{Bc}$ & - \\
$\mathbf{9}$ & $9,93 \times 10^{4} \mathrm{Ca}$ & $9,41 \times 10^{3} \mathrm{Da}$ & $2,69 \times 10^{4} \mathrm{Ba}$ & $2,29 \times 10^{3} \mathrm{Ba}$ & $9,77 \times 10^{-3} \mathrm{Bb}$ & $8,50 \times 10^{-3} \mathrm{Bb}$ & - \\
$\mathbf{1 2}$ & $1,13 \times 10^{5} \mathrm{Ba}$ & $4,27 \times 10^{4} \mathrm{Ca}$ & $7,40 \times 10^{4} \mathrm{Ba}$ & $8,44 \times 10^{3} \mathrm{Bb}$ & $2,16 \times 10^{-2} \mathrm{Ac}$ & $9,17 \times 10^{-3} \mathrm{Bc}$ & $1,21 \times 10^{-4} \mathrm{Ad}$ \\
$\mathbf{1 5}$ & $5,29 \times 10^{5} \mathrm{Ba}$ & $1,18 \times 10^{5} \mathrm{Ba}$ & $3,51 \times 10^{5} \mathrm{Aa}$ & $2,15 \times 10^{4} \mathrm{Aa}$ & $1,40 \times 10^{-2} \mathrm{Ab}$ & $2,02 \times 10^{-2} \mathrm{Ab}$ & - \\
$\mathbf{1 8}$ & $2,05 \times 10^{6} \mathrm{Aa}$ & $7,72 \times 10^{5} \mathrm{Aa}$ & $6,93 \times 10^{5} \mathrm{Aa}$ & $9,41 \times 10^{4} \mathrm{Aa}$ & $2,82 \times 10^{-2} \mathrm{Ab}$ & $2,29 \times 10^{-2} \mathrm{Ab}$ & $4,37 \times 10^{-4} \mathrm{Ac}$ \\
\hline
\end{tabular}

*Unidades formadoras de colônia por grama de amostra $\left(\right.$ UFC. $\left.^{-1}\right)$.

**T1: PEBD de 6mm; T2: AGF da Dupont de 15mm; T3: HF da Dupont de 15mm; T4: PP de 22mm.; T5: PEBD de 60mm; T6: PEBD de 80mm.; T7: PET. As médias seguidas das mesmas letras, nas linhas e nas colunas, não diferem entre si, ao nível de 5\% de probabilidade, pelo teste de Tukey. 
TABELA 3 - Contagem de bactéria lácticas, em carambolas cv. Golden Star, minimamente processadas e frigoarmazenadas por 18 dias a 12 $\pm 0,5^{\circ}$ C e U.R. $95 \pm 5 \%$.

\begin{tabular}{cccccccc}
\hline Dias & \multicolumn{7}{c}{${ }^{* *}$ Tratamentos } \\
\hline & $\mathbf{T 1}$ & $\mathbf{T 2}$ & $\mathbf{T 3}$ & $\mathbf{T 4}$ & $\mathbf{T 5}$ & T6 & T7 \\
\hline $\mathbf{1}$ & $2,27 \times 10^{-1} \mathrm{Ea}$ & $5,12 \times 10^{-1} \mathrm{Da}$ & $3,16 \times 10^{-1} \mathrm{Da}$ & $1,55 \times 10^{-1} \mathrm{Da}$ & $1,18 \times 10^{-4} \mathrm{Hb}$ & $2,26 \times 10^{-4} \mathrm{Fb}$ & - \\
$\mathbf{2}$ & $3,55 \times 10^{2} \mathrm{Da}$ & $4,72 \times 10^{1} \mathrm{Db}$ & $4,02 \times 10^{1} \mathrm{Da}$ & $8,26 \times 10^{-1} \mathrm{Dc}$ & $7,41 \times 10^{-3} \mathrm{Gd}$ & $9,44 \times 10^{-4} \mathrm{Fe}$ & - \\
$\mathbf{3}$ & $4,51 \times 10^{4} \mathrm{Ca}$ & $6,45 \times 10^{2} \mathrm{Cb}$ & $4,19 \times 10^{2} \mathrm{Cb}$ & $7,26 \times 10^{1} \mathrm{Dc}$ & $5,25 \times 10^{-2} \mathrm{Fd}$ & $4,19 \times 10^{-2} \mathrm{Ed}$ & - \\
$\mathbf{5}$ & $9,22 \times 10^{4} \mathrm{Ca}$ & $9,30 \times 10^{2} \mathrm{Cb}$ & $7,55 \times 10^{2} \mathrm{Cb}$ & $3,69 \times 10^{2} \mathrm{Cb}$ & $9,11 \times 10^{1} \mathrm{~Eb}$ & $1,33 \times 10^{2} \mathrm{Db}$ & - \\
$\mathbf{7}$ & $4,02 \times 10^{5} \mathrm{Ba}$ & $3,48 \times 10^{3} \mathrm{Bb}$ & $1,19 \times 10^{3} \mathrm{Bb}$ & $8,26 \times 10^{2} \mathrm{Cb}$ & $7,55 \times 10^{2} \mathrm{Db}$ & $8,11 \times 10^{2} \mathrm{Db}$ & - \\
$\mathbf{9}$ & $2,90 \times 10^{6} \mathrm{Aa}$ & $9,45 \times 10^{3} \mathrm{Bb}$ & $7,66 \times 10^{3} \mathrm{Bb}$ & $1,40 \times 10^{3} \mathrm{Bb}$ & $3,42 \times 10^{3} \mathrm{Cb}$ & $7,52 \times 10^{3} \mathrm{Cb}$ & - \\
$\mathbf{1 2}$ & $5,86 \times 10^{6} \mathrm{Aa}$ & $3,71 \times 10^{4} \mathrm{Ab}$ & $9,02 \times 10^{3} \mathrm{Bb}$ & $6,11 \times 10^{3} \mathrm{Bb}$ & $2,02 \times 10^{4} \mathrm{Bb}$ & $5,40 \times 10^{4} \mathrm{Bb}$ & - \\
$\mathbf{1 5}$ & $8,43 \times 10^{6} \mathrm{Aa}$ & $6,57 \times 10^{4} \mathrm{Ac}$ & $4,91 \times 10^{4} \mathrm{Ac}$ & $2,09 \times 10^{4} \mathrm{Ac}$ & $3,02 \times 10^{5} \mathrm{Ab}$ & $3,65 \times 10^{5} \mathrm{Ab}$ & - \\
$\mathbf{1 8}$ & $8,52 \times 10^{6} \mathrm{Aa}$ & $9,02 \times 10^{4} \mathrm{Ac}$ & $8,66 \times 10^{4} \mathrm{Ac}$ & $6,50 \times 10^{4} \mathrm{Ac}$ & $8,75 \times 10^{5} \mathrm{Ab}$ & $6,47 \times 10^{5} \mathrm{Ab}$ & - \\
\hline
\end{tabular}

*Unidades formadoras de colônia por grama de amostra $\left(\mathrm{UFC} . \mathrm{g}^{-1}\right)$.

**T1: PEBD de 6mm; T2: AGF da Dupont de 15mm; T3: HF da Dupont de 15mm; T4: PP de 22mm.; T5: PEBD de 60mm; T6: PEBD de 80mm.; T7: PET. As médias seguidas das mesmas letras, nas linhas e nas colunas, não diferem entre si, ao nível de 5\% de probabilidade, pelo teste de Tukey.

sanitárias impostas durante a execução do trabalho eram satisfatórias.

Entretanto, observou-se que o tratamento com o hipoclorito de sódio, a exemplo do que ocorreu com o experimento de Bonnas et al. (2003), não foi eficiente para o controle de fungos, leveduras (Tabela 2) e bactérias lácticas em alguns dos tratamentos testados (Tabela 3). No entanto, os frutos acondicionados nas embalagens PET, dada a estabilização da atmosfera gasosa no interior das embalagens em níveis médios aproximados de $7 \%$ de $\mathrm{O}_{2}$ e $8 \%$ de $\mathrm{CO}_{2}$ já nos primeiros dias de AR, não apresentaram indícios desses tipos de contaminantes ao longo do experimento (Tabelas 2 e 3 ). A inibição do crescimento de fungos e leveduras promovidas pela atmosfera modificada também foi verificada por Mohd-Som et al. (1994) (citado por Pereira et al., 2003) em brócolis minimamente processados, mostrando que elevados níveis de $\mathrm{CO}_{2}$ e baixos níveis de $\mathrm{O}_{2}$ apresentam efeitos benéficos na inibição desses microrganismos. Pereira et al. (2003) somente relatam o efeito positivo no controle de fungos, leveduras e bactérias lácticas à associação entre a embalagem PET e a desidratação osmótica das goiabas minimamente processadas, descartando, por sua vez, a eficácia do uso da atmosfera modificada em frutos in natura. Ao contrário do observado neste trabalho, cujas carambolas minimamente processadas, sem nenhum tratamento complementar e acondicionadas em embalagens PET, não apresentaram quaisquer índices representativos de contaminação microbiológica.

Já nos frutos embalados nos filmes de PEBD de 0,060 e 0,080 $\mathrm{mm}$, a presença de fungos e leveduras (Tabela 2) não foi expressiva, variando de $1,21 \times 10^{-3} \mathrm{UFC} . \mathrm{g}^{-1}$ no primeiro dia a $2,82 \times 10^{-2} \mathrm{UFC}^{-g^{-1}}$ ao final dos 18 dias de AR. Nesses tratamentos, a partir do $12^{0}$ dia de $\mathrm{AR}$, quando as concentrações de $\mathrm{CO}_{2}$ estabilizaram em níveis mais elevados, a contagem de bactérias estava aproximadamente em 5,4 $\mathrm{x}$ $10^{4} \mathrm{UFC} \mathrm{g}^{-1}$ (Tabela 4), proporcionando a manifestação de aromas e sabores desagradáveis (dados não publicados). Estes sintomas podem representar, segundo Neves et al. (2004), o efeito direto da fermentação do produto, supostamente devido às condições da atmosfera gasosa no interior das embalagens a partir do $12^{\underline{0}}$ dia de AR. O comportamento acima citado, possivelmente, seja devido ao controle tardio da estabilização dos níveis de $\mathrm{CO}_{2}$ e $\mathrm{O}_{2}$ no interior dessas embalagens, tendo então potencializado, a partir do $12^{\circ}$ dia de AR, condições favoráveis ao crescimento das bactérias lácticas. Nos tratamentos acondicionados em filmes de PEBD de 0,006 mm perfurados e AGF e HF de 0,015 mm e PP de 0,022 mm, observou-se que, a partir do $5^{\circ}$ dia de $\mathrm{AR}$, a contagem de fungos e leveduras variava entre $3,77 \times 10^{2} \mathrm{UFC} . \mathrm{g}^{-1}$ e $1,24 \times 10^{3} \mathrm{UFC} . \mathrm{g}^{-1}$, ao passo que, aos 15 dias de AR, a contagem havia subido para valores entre 2,15 x $10^{4}$ UFC. $^{-1}$ e 5,29 x $10^{5}$ UFC. $^{-1}$ (Tabela 2). Da mesma maneira, na contagem de bactérias lácticas, desde o terceiro dia de $\mathrm{AR}$, os números estavam entre 7,26 x $10^{1} \mathrm{e} 4,51 \times 10^{4} \mathrm{UFC}^{-\mathrm{g}^{-1}}$ (Tabela 3 ).

Características físico-químicas - As embalagens testadas interagiram diferentemente na manutenção dos atributos qualitativos das carambolas minimamente processadas (Tabela 1). Nesse sentido, observa-se que somente nas análises de SS e AT é que os tratamentos não apresentaram diferença significativa entre si. Desta forma, mesmo não apresentando padrão climatérico (Neves et al., 2004), os pedaços de carambolas cv. Golden Star apresentaram pequeno e gradativo aumento nos teores de SS, não apresentando, entretanto, alterações significativas em função dos tratamentos testados. $\mathrm{O}$ pequeno e não-significativo incremento detectado nos teores de SS, provavelmente, deve-se tão somente à desidratação parcial desses pedaços de carambola durante o período de AR, concentrando, portanto, o conteúdo celular. Lamikanra et al. (2000) também não observaram mudanças significativas nos teores de SS em melões cv. Cantaloupe minimamente processados mantidos a $4^{\circ} \mathrm{C}$ por 14 dias.

Quanto aos níveis de AT, constataram-se decréscimos progressivos e acentuados, porém não-significativos, a exemplo da análise de SS, entre os tratamentos testados. AAT que se apresentava com níveis médios de 13,9 Cmol. $\mathrm{L}^{-1}$ no momento da colheita, alcançou níveis médios de 9,6 $\mathrm{Cmol}^{-1}$ aos 9 dias de $\mathrm{AR}$, chegando ao final do experimento com níveis médios de $6,8 \mathrm{Cmol} . \mathrm{L}^{-1}$. Esse comportamento indica que não houve influência significativa da $\mathrm{AM}$, através dos diferentes tipos de embalagens testadas, no metabolismo de transformação dos ácidos orgânicos durante o AR. Yamashita et al. (2001), trabalhando com mangas cv. Tommy Atkins, não observaram influência da AM na manutenção dos níveis de AT dos frutos armazenados in natura. No entanto, Bonnas et al. (2003) verificaram em abacaxis minimamente processados, acondicionados em embalagens de PVC e PP (sem detalhamento), a diminuição na velocidade das perdas de AT, refletindo, por sua vez, na melhor qualidade ao final do período de armazenamento. Deste modo, podese condicionar o efeito da AM em relação à AT, ao tipo de embalagem, taxa de permeabilidade, grau de processamento dos frutos e às características individuais de cada espécie.

A partir da análise do $\mathrm{pH}$, pôde-se estabelecer um paralelo comparativo entre os resultados microbiológicos, anteriormente discutidos (Tabelas 2 e 3), com as variações de pH (Tabela 1). Nos tratamentos acondicionados em filmes de PEBD de 0,006 mm perfurados e AGF e HF de $0,015 \mathrm{~mm}$, nos quais foram observados os maiores índices de contaminações microbiológicas, o pH apresentouse relativamente elevado. Nesses tratamentos, o pH variou em média de 3,51 na colheita, para 4,98 aos 5 dias de AR, chegando a 7,21 aos 18 dias de AR. Partindo-se então do pressuposto de que a manutenção do $\mathrm{pH}$, em níveis mais baixos, consista em um importante fator de restrição ao crescimento de microrganismos nos alimentos (Antoniolli et al., 2001), o elevado pH observado nesses tratamentos, pode ter influência direta nos elevados índices de contaminação microbiológica desses frutos, ou, até mesmo, ser um reflexo da intensa 
atividade microbiana. Nos tratamentos acondicionados em embalagens de PEBD de 0,060 e 0,080 mm, o comportamento do $\mathrm{pH}$ apresentou dois padrões distintos. Da colheita até os 12 dias de AR, o pH sofreu gradativo aumento, não apresentando, no entanto, sinais de contaminação microbiológica expressiva. Porém, após o $12^{\circ}$ dia de AR, quando a composição gasosa teria se estabelecido em um patamar favorável (Figura 1), o pH alcançou níveis médios de 5,50 aos 15 dias de AR, chegando ao final do experimento com níveis não inferiores a 6,91. Esse comportamento pode então comprovar que a estabilização tardia da atmosfera gasosa nessas embalagens não proporcionou benefícios qualitativos. Neste caso, o aumento de $\mathrm{pH}$, associado à baixa permeabilidade gasosa, pode ter desencadeado o desenvolvimento de bactérias lácticas, o que, por suas vez, contribuiu diretamente para a perda de qualidade do produto final.

Já nos frutos acondicionados em embalagens PET, o pH apresentou as menores variações dentre os tratamentos testados, ou seja, variou em média de 3,51 no momento da colheita, para 4,26 aos 18 dias de AR. Nesse sentido, é possível validar a afirmação de Antoniolli et al. (2001), em que a manutenção do pH, em níveis mais baixos, pode consistir em uma ferramenta eficiente para o controle microbiano.

Quanto ao teor de ácido ascórbico nas carambolas minimamente processadas, os maiores valores foram detectados nas carambolas acondicionadas nas embalagens PET (Tabela 1). Esses pedaços continham, em média, no momento da colheita, 19,02 mg de ácido ascórbico. $100 \mathrm{~g}^{-1}$ de polpa, chegando, ao final de 18 dias de $\mathrm{AR}$, com valores médios de $2,95 \mathrm{mg}$ de ácido ascórbico. $100 \mathrm{~g}^{-1} \mathrm{de}$ polpa. O mesmo foi relatado por Antoniolli et al. (2001) observando reduções nos teores de ácido ascórbico em abacaxis minimamente processados. Em relação aos demais tipos de embalagens testadas, onde o controle da atmosfera gasosa foi totalmente ineficiente ou tardia, não foram observadas diferenças significativas entre si, apresentando perdas variando entre 98 a $100 \%$ frente ao conteúdo inicial. Assim, verificaram-se valores entre 0,37 mg de ácido ascórbico. $100 \mathrm{~g}^{-1}$ de polpa à ausência absoluta de ácido ascórbico nos pedaços de carambola. Esses resultados podem ser considerados como pertinentes, principalmente partindo-se do propósito de que esse tipo de substância apresenta grande sensibilidade ao $\mathrm{O}_{2}$.

\section{CONCLUSÕES}

Com base nos resultados obtidos neste experimento, recomenda-se o uso de bandejas rígidas de polietileno tereftalato (PET), com capacidade para $500 \mathrm{~mL}$, com tampa do mesmo material, para a concepção da atmosfera modificada passiva, em carambolas minimamente processadas. Deste modo, estima-se uma vida útil desse produto de até 18 dias em atmosfera refrigerada, com boa manutenção dos atributos físico-químicos e microbiológicos.

\section{REFERÊNCIAS}

AGÊNCIA NACIONAL DE VIGILANCIA SANITÁRIA. Resolução RCD n. 12, de 2 de janeiro de 2001. Disponível em: <http:// www.anvisa.gov.br.2001>.

AHVENAINEN, R. New approaches in improving the shelf-life of minimally processed fruit and vegetables. Trends in Food Science \& Tecnology, Amsterdam, v. 7, n. 6, p. 179-187, 1996.

ANTONIOLLI, L.R.; CASTRO, P.R.de C.; KLUGE, R.A.; FILHO, J.A.S. Influência da embalagem de polietileno na remoção da adstringência e na qualidade de caquis (Diospyrus kaki L.), cv. Giombo, armazenados sob refrigeração. Revista Brasileira de Fruticultura, Jaboticabal, v.23, n.2, p.293-297, 2001.
ARRUDA, M.C.; JACOMINO, A.P.; SARANTÓPOULOS, C.I.G.L.; MORETTI, C.I. Qualidade de melão minimamente processado armazenado em atmosfera modificada passiva. Horticultura Brasileira, Brasília, v.21, n.4, 2003.

BONNAS, S.D.; CHITARRA, A.B.; PRADO, M.E.T.; JÚNIOR, D.T. Qualidade do abacaxi cv. Smooth Cayenne minimamente processado. Revista Brasileira de Fruticultura, Jaboticabal, v. 25, n.2, p. 206-209, 2003.

BRACKMANN, A.; STEFFENS, C.A.; GIEHL, R.F.H. Armazenamento de pêssego 'Chimarrita' em atmosfera controlada e sob absorção de etileno. Ciência Rural, Santa Maria, v.33, n.3,p 119-124, 2003.

CANTWELL, M. Postharvest handling systems: minimally processed fruits and vegetables. In: CARVALHO, C. R. L.; MANTOVANI, D. M. B.; CARVALHO, P. R. N.; MORAES, R. M. M. Análises químicas de alimentos: manual técnico. Campinas: Instituto de Tecnologia de Alimentos, 1990. 121p.

CARVALHO, C. R. L.; MANTOVANI, D. M. B.; CARVALHO, P. R. N.; MORAES, R. M. M. Análises químicas de alimentos: manual técnico. Campinas: Instituto de Tecnologia de Alimentos, 1990. $121 \mathrm{p}$.

DONADIO, L.C.; SILVA, J.A.A.; ARAÚJO, P.R.S.; PRADO, R.M. Caramboleira (Averrhoa carambola L.). Jaboticabal: Sociedade Brasileira de Fruticultura, 2001. 81p. (Série Frutas Potenciais).

DONAZOLLO, J.; HUNSCHE, M.; BRACKMANN, A.; WACLAWOVSKY, A.J. Utilização de filmes de polietileno de baixa densidade (PEBD) para prolongar a vida pós-colheita de morangos, cv. Oso Grande. Ciência Agrotécnica, Lavras. V.27, n.1, p.165-172, 2003.

HONG, S.; KIM, D. Influence of oxygen concentration and temperature on respiratory characteristics of fresh-cut green onion. International Journal of Food Science and Technology, Oxford, v. 36, p. 283-289, 2001.

JUNQUEIRA, A.H.; LUENGO, R.F.A. Mercado diferenciados de hortaliças. Horticultura Brasileira, Brasília, v. 18, n.2, p.134$137,2000$.

LAMIKANRA, O.; CHEN, J.C.; BANKS, D. Biochemical and microbial changes during the storage of minimally processes cantaloupe. Journal of Agricultural and Food Chemistry, Washington, v.48, p. 5.955-5.961, 2000.

LANA, M.M.; FINGER, F.L. Atmosfera modificada e controlada. Aplicação na conservação de produtos hortícolas. Brasília: Embrapa Hortaliças, 2000.34p.

MOHD-SOM, F.; KERBEL, E.; MARTIN, S.E.; SCHMIDT, S.J. Microflora Changes in Modified Atmosphere- Packanged Broccoli Florets Stores at Refrigerated Temperature. Journal of Food Quality, Trumbull, v. 17, p. 347-360, 1994.

NEVES, L.C.; BENDER, R.J.; ROMBALDI, C.V.; VIEITES, R.L. Armazenagem em atmosfera modificada passiva de carambola azeda (Averrhoa carambola L.) cv. 'Golden Star'. Revista Brasileira de Fruticultura, Jaboticabal, v.26, n.1, 2004.

OLIVEIRA, L.M.; ALVES, R.M.V.; SARANTÓPOULOS, C.I.G.L. Ensaios para avaliação de embalagens plásticas flexíveis. Campinas: ITAL, CETEA, 1996.219 p.

PEREIRA, L.M.; RODRIGUES, A.C.C.; SARANTÓPOULOS, C.I.G.de L. Vida-de-prateileira de goiabas minimamente processadas acondicionadas em embalagens sob atmosfera modificada. Ciência e Tecnologia de Alimentos, Campinas, v. 23, n.3, p. 427433, 2003.

VANDERZANTE, C.; SPLITTSTOESSER, D.F. Compendium of methods for the microbiological examination of foods. Washington: American Public Health Association, 1992.

YAMASHITA, F.; MIGLIORANZA, L.H. da S.; MIRANDA, L. de A.; SOUZA, C.M. de A. Effects of packaging and temperature on postharvest of atemoya. Revista Brasileira de Fruticultura, Jaboticabal, v.24, n.3, p.658-660, 2002. 